\title{
CT findings of pulmonary nocardiosis: a report of 9 cases
}

\author{
Baoliang Liu ${ }^{1}$, Yuanlong Zhang ${ }^{1,2}$, Jingshan Gong ${ }^{2}$, Shubing Jiang ${ }^{1}$, Yunkai Huang ${ }^{1}$, Lingwei Wang ${ }^{3}$, \\ Jianmin $\mathrm{Xu}^{2}$, Chen Qiu ${ }^{3}$
}

${ }^{1}$ Department of Radiology, the Third People's Hospital of Shenzhen Longgang District, Shenzhen 518115, China; ${ }^{2}$ Department of Radiology, ${ }^{3}$ Department of Respiratory and Critical Medicine, Shenzhen People's Hospital, Second Clinical Medical College, Jinan University, Shenzhen 518020, China

Contributions: (I) Conception and design: J Gong; (II) Administrative support: J Xu, C Qiu; (III) Provision of study materials or patients: Y Zhang, L Wang; (IV) Collection and assembly of data: B Liu, Y Zhang; (V) Data analysis and interpretation: J Gong, S Jiang, Y Huang; (VI) Manuscript writing: All authors; (VII) Final approval of manuscript: All authors.

Correspondence to: Dr. Jingshan Gong. Department of Radiology, Shenzhen People's Hospital, Second Clinical Medical College, Jinan University, Shenzhen 518020, China. Email: jshgong@sina.com.

Background: Pulmonary nocardiosis often occurs among immunocompromised patients, especially in those with impaired cell immunity related to AIDS and transplant. As the number of patients receiving transplant, the occurrence of pulmonary nocardiosis may also increase. Radiologic characteristics of this entity have not been well documented. In present study, CT findings of clinical confirmed pulmonary nocardiosis were retrospectively reviewed in order to understand CT features of pulmonary nocardiosis.

Methods: Electronic databases of Shenzhen People's Hospital, Shenzhen, China, and the Third People's Hospital of Longgang District, Shenzhen, China were searched for patients with definitive diagnosis of pulmonary nocardiosis and available CT images. Nine patients were founded and they were included in this retrospective analysis. Clinical presentations at diagnosis and related medical history were recorded. CT images were retrospectively reviewed by two radiologists in consensus.

Results: The patients consisted of seven men and two women with a median age of 47 years (range, 20-73 years). There were three immunocompromised patients and six immunocompetent patients. The latter included two patients had history of bronchiectasis. The symptoms at the diagnosis were nonspecific of fever, cough, sputum, dyspnea, chest pain, and hemoptysis. CT findings included: lung consolidation $(n=8)$ with cavitation in three cases and foci of decreased enhancement in one patient who had contrast-enhanced CT, nodules/masses $(n=6)$ with cavitation in two patients, ground-glass opacity $(n=4)$, centrilobular nodules $(n=4)$, pleural effusion $(n=4)$, bronchiectasis $(n=4)$, interlobular septal thickening $(n=3)$, interlobular reticular opacity ( $n=3)$, lymph node enlargement ( $n=2)$, halo sign $(n=2)$ and mucoid impaction $(n=1)$.

Conclusions: Lung consolidation and nodules/masses are the most common findings, but CT findings of pulmonary nocardiosis are diverse and nonspecific. In some clinical background, such as immunocompromised patients or with some underlying lung diseases, consolidation or nodules/masses with cavitation may suggest the possibility of pulmonary nocardiosis.

Keywords: Nocardiosis; pulmonary; computed tomography (CT)

Submitted Sep 09, 2017. Accepted for publication Sep 19, 2017.

doi: $10.21037 /$ jtd.2017.09.122

View this article at: http://dx.doi.org/10.21037/jtd.2017.09.122

\section{Introduction}

Nocardia is a genus of filamentous gram-positive, weakly acid fast, aerobic bacteria found in dust, soil, water, decaying vegetation, and stagnant matter (1). Lung infection can occur through direct inhalation of nocardia, which accounts for $73-77 \%$ of infected patients (2). Although pulmonary nocardiosis often occurs in immunocompromised patients, especially in those with impaired cell immunity related to AIDS and transplant, one-third of patients with nocardiosis 
have normal immunity $(3,4)$.

Because its clinical presentations are nonspecific as subacute pneumonia, pulmonary nocardiosis is difficult to diagnose. If correct diagnosis can be obtained, pulmonary nocardiosis respond favorably to high-dose sulfonamide therapy, otherwise, hematogenous dissemination to soft tissue and brain is association with high morbidity and mortality (5-7). Therefore, prompt diagnosis and treatment is essential for improving patients' outcome.

As the growing number of immunosuppressed patients, especially those with impaired cell-mediated immunity related to AIDS and transplant, infections of nocardia may increase also (8). In United States, nocardia infections occurred in about $22 \%$ of patients receiving organ transplant (9). Nocardia most often enters the hosts through respiratory tracts and produces infection in the lungs, although isolated brain soft tissue abscess have been reported (10). Imaging findings of pulmonary nocardiosis are not well documented. Only a few reports described CT findings of pulmonary nocardiosis, which were varied and nonspecific, including nodules/masses, ground-glass opacity, interlobular septal thickening, cavitation, and pleural effusion (9,11-15). Here we reported nine patients with pulmonary nocardiosis to evaluate its CT features.

\section{Methods}

Electronic databases of Shenzhen People's Hospital, Shenzhen, China, and the Third People's Hospital of Shenzhen Longgang District, Shenzhen, China, were searched for patients with definitive diagnosis of pulmonary nocardiosis and available CT images. This study was approved by the institutional review boards and the requirement for informed consent was waived due to its retrospective character. Clinical presentations at diagnosis and related medical history were recorded.

Thoracic CT examinations were performed at a Philips Brilliance 16 (Philips Medical Systems) or a Siemens SOMATOM Definition AS64 (Siemens Medical Solutions). The parameters were as follows: detector configuration, $16 \mathrm{~mm} \times 1.5 \mathrm{~mm}$ or $64 \mathrm{~mm} \times 0.625 \mathrm{~mm}$; tube voltage, 120 to $140 \mathrm{kVp}$; tube current, 200 to $240 \mathrm{~mA}$. Two sets of images were constructed: $5.0 \mathrm{~mm}$ slice thickness with $5.0 \mathrm{~mm}$ gap and $2.0 \mathrm{~mm}$ slice thickness with $1.0 \mathrm{~mm}$ gap. The latter was used for multiple planar reconstruction. Follow-up CT examinations were performed at seven patients. Contrastenhanced CT was performed two patients. The CT images were evaluated by two radiologists in consensus on picture archiving and communication system (PACS).

\section{Results}

\section{Patients demographics}

The patients consisted of seven men and two women with a median age of 47 years (range, 20-73 years). There were three immunocompromised patients and six immunocompetent patients. Among the six immunocompetent patients, two patients had history of bronchiectasis. The symptoms at the diagnosis of pulmonary nocardiosis included fever $(\mathrm{n}=5)$ and cough $(n=4)$, sputum $(n=3)$, dyspnea $(n=2)$, chest pain $(n=2)$ and hemoptysis $(\mathrm{n}=2)$.

\section{CT findings}

All the nine patients had abnormalities on thoracic CT, which were summarized in Table 1 . The most common CT findings were consolidation $(\mathrm{n}=8,88.9 \%)$ and nodules/masses $(\mathrm{n}=6$, $66.7 \%)$. Cavitation could be seen at consolidation in three cases (Figure 1) and at nodules/masses in two cases (Figure 2). Foci of decreased enhancement were noted at consolidation in one patient who had contrast-enhanced CT (Figure 3). Some CT features were illustrated in Figures 4-7. After a definitive diagnosis was obtained and prompt treatment, CT abnormalities in the seven patients who had follow-up CT were improved (disappeared or decreased) (Figure 8).

\section{Discussion}

Definitive diagnosis of pulmonary nocardiosis bases on histopathologic examination or culture. Unfortunately, difficulty in organism isolation and slow growth often retard prompt diagnosis. The clinicians often look for radiographic clues to the diagnosis of pulmonary nocardiosis. In the present nine-patient series, we found that the most common CT findings of pulmonary nocardiosis were lung consolidation and nodules/masses, followed by groundglass opacity, centrilobular nodules, pleural effusion, bronchiectasis, interlobular septal thickening, interlobular reticular opacity, lymph node enlargement, halo sign and mucoid impaction. Cavitation or foci of decreased enhancement could be seen in consolidation and nodules/ masses. To best of our knowledge, several findings, such as centrilobular nodules, interlobular reticular opacity, lymph node enlargement and mucoid impaction, had not been reported before. After treatment, the abnormalities showed 
Table 1 CT findings of patients with pulmonary nocardiosis

\begin{tabular}{lcccccccccc}
\hline Variables & Case 1 & Case 2 & Case 3 & Case 4 & Case 5 & Case 6 & Case 7 & Case 8 & Case 9 \\
\hline Consolidation & $(+)$ & $(+)$ & $(-)$ & $(+)$ & $(+)$ & $(+)$ & $(+)$ & $(+)$ & $(+)$ \\
Nodules/masses & $(+)$ & $(+)$ & $(-)$ & $(+)$ & $(+)$ & $(-)$ & $(+)$ & $(-)$ & $(+)$ \\
Ground-glass opacity & $(+)$ & $(-)$ & $(-)$ & $(+)$ & $(+)$ & $(-)$ & $(-)$ & $(-)$ & $(+)$ \\
Centrilobular nodules & $(+)$ & $(-)$ & $(-)$ & $(-)$ & $(+)$ & $(-)$ & $(-)$ & $(+)$ & $(+)$ \\
Pleural effusion & $(+)$ & $(-)$ & $(+)$ & $(-)$ & $(-)$ & $(+)$ & $(+)$ & $(-)$ & $(-)$ & $(+)$ \\
Bronchiectasis & $(+)$ & $(-)$ & $(-)$ & $(-)$ & $(+)$ & $(-)$ & $(-)$ & $(+)$ & $(-)$ \\
Interlobular septal & $(+)$ & $(-)$ & $(-)$ & $(-)$ & $(+)$ & $(+)$ & $(-)$ & $(-)$ & $(-)$ \\
thickening & & & & & & & & & \\
Lymph node & $(-)$ & $(-)$ & $(-)$ & $(+)$ & $(-)$ & $(-)$ & $(-)$ & $(-)$ & $(+)$ \\
enlargement & $(-)$ & $(+)$ & $(-)$ & $(-)$ & $(-)$ & $(-)$ & $(-)$ & $(-)$ & $(+)$ \\
Halo sign & $(-)$ & $(-)$ & $(-)$ & $(+)$ & $(-)$ & $(-)$ & $(-)$ & $(-)$ & $(-)$ \\
Mucoid impaction & & & & & & & & & & \\
\hline
\end{tabular}

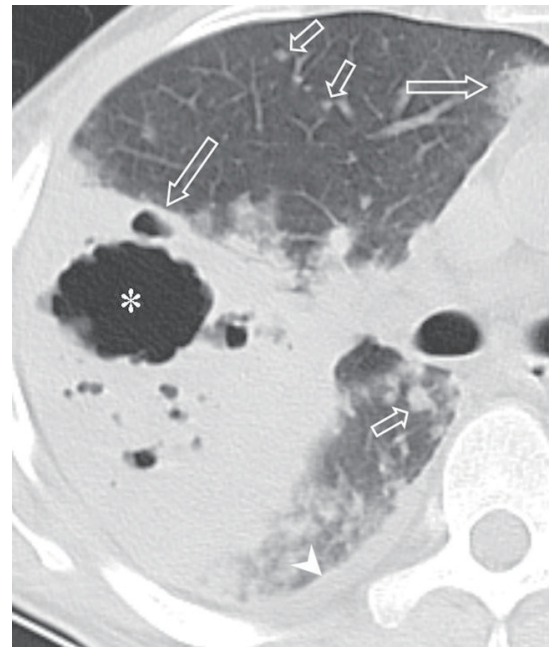

Figure 1 Pulmonary nocardiosis in a 21-year-old immunocompetent male. Transverse CT image shows consolidations (long arrows), cavitation (star), nodules (short arrows) and pleural effusion (arrow head) in the right lung. Cavitation (star) can be seen in one consolidation.

disappeared or decreased.

Pulmonary nocardiosis is the most common type, which consists of $73-77 \%$ of nocardia infections (2). However, only several case reports deal with radiologic findings of pulmonary nocardiosis. Radiographic findings of pulmonary nocardiosis included consolidation with or without cavitation, nodules, pleural effusion, and chest wall extension, regardless

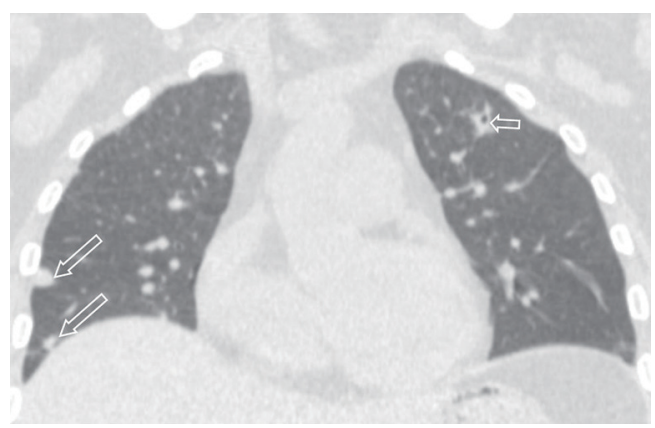

Figure 2 Pulmonary nocardiosis in a 21-year-old immunocompetent male. Coronal CT image shows multiple nodules (long arrows and short arrow). Cavitation can be seen in one nodule (short arrow).

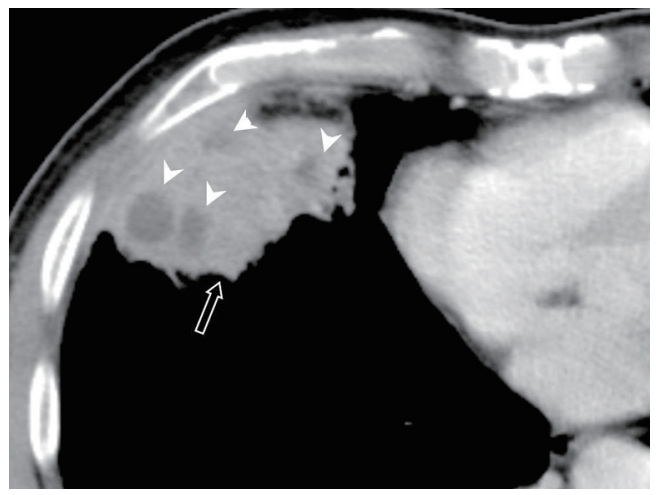

Figure 3 Pulmonary nocardiosis in a 58-year-old immunocompetent male. Transverse contrast-enhanced CT image shows a heterogeneous enhanced mass (arrow) with several low enhanced areas (arrows). 

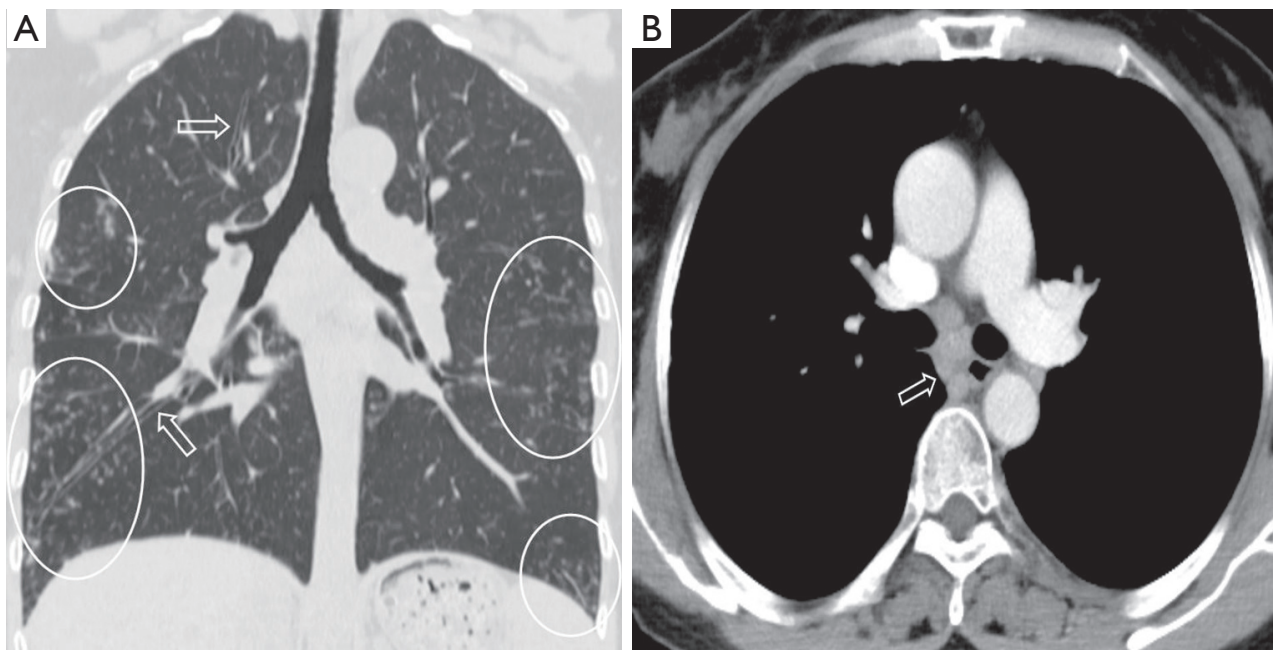

Figure 4 Pulmonary nocardiosis in a 54-year-old immunocompetent female with 2-year-history of bronchiectasis. (A) Coronal CT image shows centrilobular nodules (circles) and bronchial wall thickening (arrows); (B) transverse contrast-enhanced CT image demonstrates lymph node enlargement (arrow).

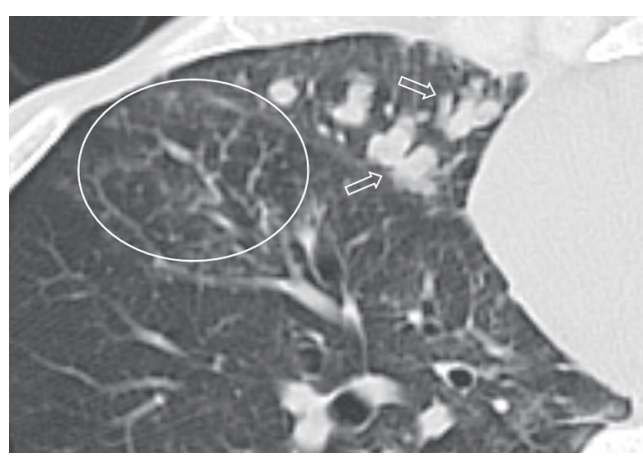

Figure 5 Pulmonary nocardiosis in a 47-year-old male with nasopharyngeal carcinoma. Transverse CT image shows mucoid impaction (arrows) and centrilobular nodules (circle).

of underlying etiology $(16,17)$. High resolution CT (HRCT), which can depict lung structures and pathogenic changes with excellent details, is often used to evaluate pulmonary parenchyma and interstitial abnormalities (18-20). Comparing to conventional radiography, HRCT can detect pathologic changes of pulmonary nocardiosis at early phase and in better characteristics $(5,6)$. In a pictorial review, Kanne $e t$ al. illustrated that CT findings of pulmonary nocardiosis included lung consolidation, nodules/masses, pleural involvement and chest wall extension (9). Oszoyoglu and colleagues reported the most common abnormalities on CT were nodules (71\%) and cavitary lesions (43\%) without any significant zonal or anatomic distribution in pulmonary

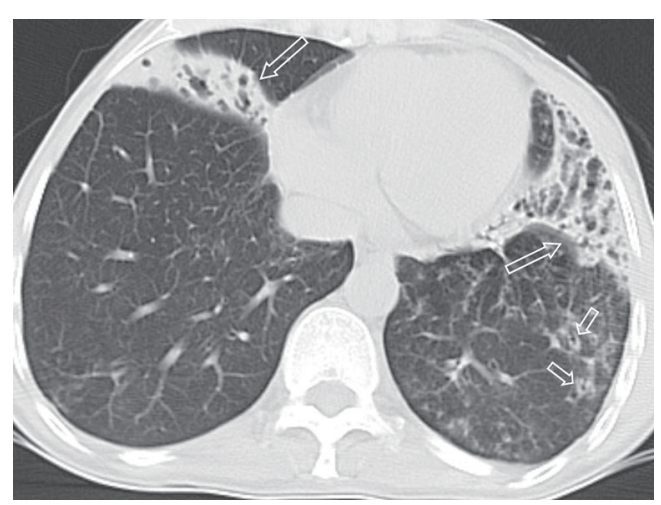

Figure 6 Pulmonary nocardiosis in a 46-year-old female with 20-year-history of bronchiectasis. Transverse CT image shows consolidations with bronchiectasis (long arrows) and bronchial wall thickening (short arrows).

nocardiosis after lung transplantation (13). Sato et al., respectively, analyzed HRCT of 18 patients with pulmonary nocardiosis (14). The most common findings were a nodule/ mass $(94.4 \%)$, ground-glass opacity $(77.8 \%)$, interlobular septal thickening $(77.8 \%)$, and cavitation $(66.7 \%)$. What we had found in present series was similar to the literatures, but we also noted some abnormalities had not been reported before.

For nocardia spreads in respiratory tracts, colonizes there and causes bronchopneumonia. Pathological findings of bronchopneumonia consist of inflammatory changes 
in bronchial and bronchiolar walls, as well as exudation into adjacent alveoli. Therefore, bronchial wall thickening and centrilobular nodules are frequently found in patients with bronchopneumonia, sometimes forming tree-inbud sign and mucoid impaction. Except bronchial wall thickening and interlobular septal thickening had reported before, centrilobular nodulars and mucoid impaction was not motioned in the literatures. In present series, centrilobular nodules and mucoid impaction were seen in

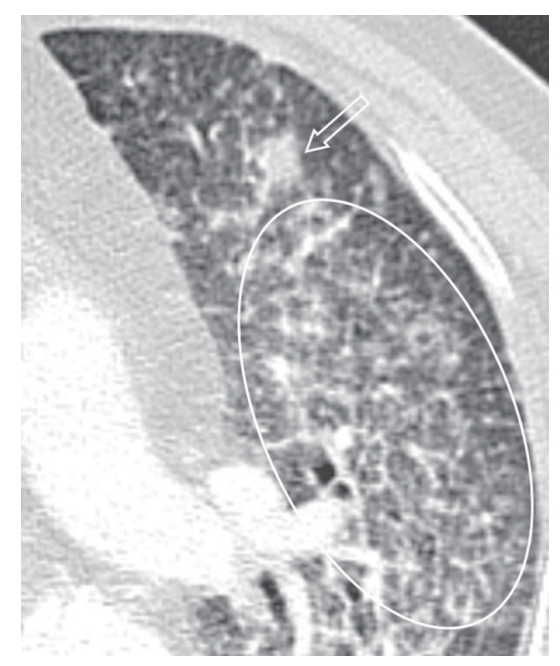

Figure 7 Pulmonary nocardiosis in a 73-year-old immunocompetent male. Transverse CT image shows a patchy consolidation (arrow) and interlobular reticular opacity (circle). four patients and one patient, respectively. Besides these two signs, interlobular reticular opacity and lymph node enlargement were not reported before. One explanation is that CT features of pulmonary nocardiosis has not been well documented and might be different in different areas or clinical background. Another reason perhaps is that other infections might be concurrent in these patients. Therefore, some abnormalities might result from other infections.

It was reported that preexisting structural lung diseases, such as bronchiectasis and pulmonary alveolar proteinosis, could increase the risk of nocardia colonization. In present series, there were two patients with history of bronchiectasis, and bronchiectasis was identified in four patients at the diagnosis of pulmonary nocardiosis. Therefore, some underlying lung pathological changes may provide colonizing sites for nocardia.

This retrospective study only included nine patients with pulmonary nocardiosis. The small sample size prevented to conclude specific radiographic clues for pulmonary nocardiosis and to compare CT findings between immunocompetent and immunocompromised patients. Another limitation of the study was that concurrent other infections were not excluded in all the patients. Therefore, some abnormalities might not result from nocardia infection in this series. Third, although we reported several CT findings firstly in pulmonary nocardiosis, histopathological correlation was not performed.

In summary, pulmonary nocardiosis can occur in both immunocompromised and immunocompetent patients.
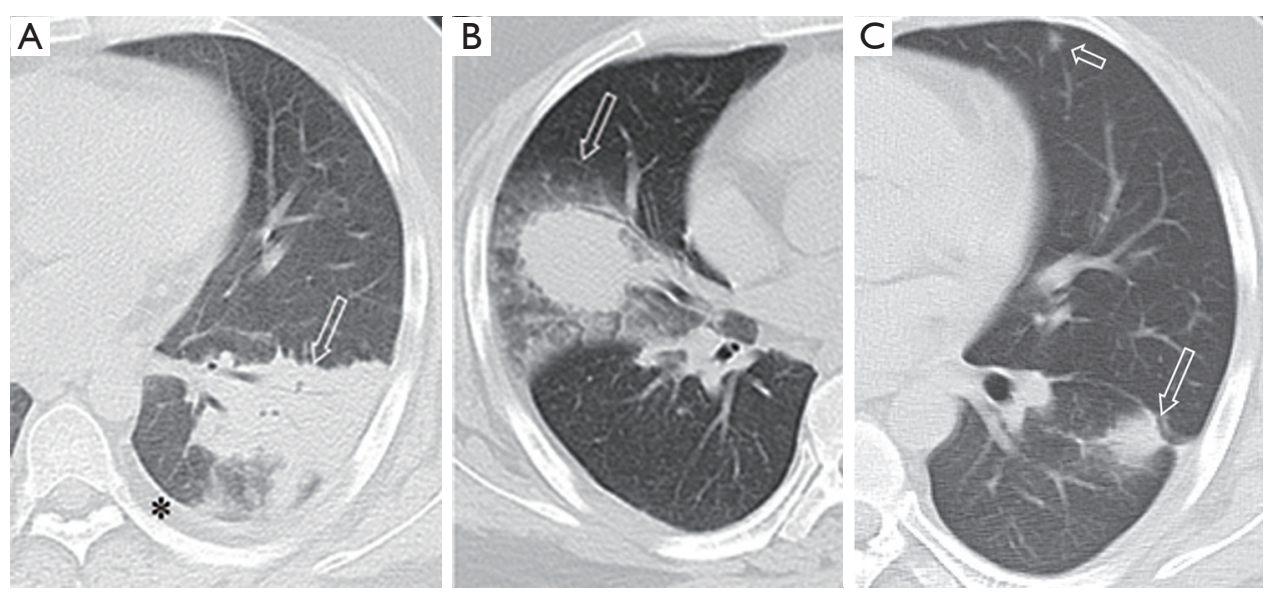

Figure 8 Pulmonary nocardiosis in a 20-year-old male with nephritic syndrome and iatrogenic Cushing syndrome. (A) transverse CT image shows a consolidation (arrow) and pleural effusion (star); (B) transverse CT image of other slice shows a mass with halo sign (arrow); (C) transverse CT image at similar level of A after treatment show the consolidation (long arrow) on A decreased. A small patchy consolidation (short arrow) is noted also. 
Lung consolidation and nodules/masses with or without cavitation are the most common findings, but CT findings of pulmonary nocardiosis are diverse and nonspecific. In some clinical background, such as immunocompromised patients or with some underlying lung diseases, consolidation or nodules/masses with cavitation may suggest the possibility of pulmonary nocardiosis. Acknowledge of CT findings can help radiologists to include pulmonary nocardiosis into differential diagnosis, and facilitate clinicians' further diagnostic and treatment decision making, especially in those immunocompromised patients or with underlying diseases.

\section{Acknowledgements}

None.

\section{Footnote}

Conflicts of Interest: The authors have no conflicts of interest to declare.

Ethical Statement: This study was approved by the institutional review boards and the requirement for informed consent was waived due to its retrospective character.

\section{References}

1. McNeil MM, Brown JM. The medically important aerobic actinomycetes: epidemiology and microbiology. Clin Microbiol Rev 1994;7:357-417.

2. Batista MV, Pierrotti LC, Abdala E, et al. Endemic and opportunistic infections in Brazilian solid organ transplant recipients. Trop Med Int Health 2011;16:1134-42.

3. Beaman BL, Burnside J, Edwards B, et al. Nocardia infection in the United States, 1972-1974. J Infect Dis 1976;134:286-9.

4. Lederman ER, Crum NF. A case series and focused review of nocardiosis: clinical and microbiologic aspects. Medicine 2004;83:300-13.

5. Husain S, McCurry K, Dauber J, et al. Nocardia infection in lung transplant recipients. J Heart Lung Transplant 2002;21:354-9.

6. Wang HK, Sheng WH, Hung CC, et al. Clinical characteristics, microbiology, and outcomes for patients with lung and disseminated nocardiosis in a tertiary hospital. J Formos Med Assoc 2015;114:742-9.

7. Blackmon KN, Ravenel JG, Gomez JM, et al. Pulmonary nocardiosis computed tomography features at diagnosis. J Thorac Imaging 2011;26:224-9.

8. Filice GA. Nocardiosis in persons with human immunodeficiency virus infection, transplant recipients, and large, geographically defined populations. J Lab Clin Med 2005;145:156-62.

9. Kanne JP, Yandow DR, Mohammed TL, et al. CT findings of pulmonary nocardiosis. AJR Am J Roentgenol 2011;197:W266-72.

10. Beaman BL, Beaman L. Nocardia species: host-parasite relationships. Clin Microbiol Rev 1994;7:213-64.

11. Yoon HK, Im JG, Ahn JM, et al. Pulmonary nocardiosis: CT findings. J Comput Assist Tomogr 1995; 19:52-5.

12. Buckley JA, Padhani AR, Kuhlman JE. CT features of pulmonary nocardiosis. J Comput Assist Tomogr 1995;19:726-32.

13. Oszoyoglu AA, Kirsch J, Mohammed TL. Pulmonary nocardiosis after lung transplantation: CT findings in 7 patients and review of the literature. J Thorac Imaging 2007;22:143-8.

14. Sato H, Okada F, Mori T, et al. High-resolution Computed Tomography Findings in Patients with Pulmonary Nocardiosis. Acad Radiol 2016;23:290-6.

15. Tsujimoto N, Saraya T, Kikuchi K, et al. High-resolution CT findings of patients with pulmonary nocardiosis. J Thorac Dis 2012;4:577-82.

16. Georghiou PR, Blacklock ZM. Infection with Nocardia species in Queensland: a review of 102 isolates. Med J Aust 1992;156:692-7.

17. Ambrosioni J, Lew D, Garbino J. Nocardiosis: update clinical review and experience at a tertiary center. Infection 2010;38:89-97.

18. Bahtouee M, Saberifard J, Nabipour I, et al. Combined computed tomography (CT)/scintigraphy strategy may help in diagnostic dilemmas in interstitial lung disease (ILD). Quant Imaging Med Surg 2016;6:460-1.

19. Dennie C, Thornhill R, Sethi-Virmani V, et al. Role of quantitative computed tomography texture analysis in the differentiation of primary lung cancer and granulomatous nodules. Quant Imaging Med Surg 2016;6:6-15.

20. Souza CA, Müller NL, Flint J, et al. Idiopathic pulmonary fibrosis: spectrum of high-resolution CT findings. AJR Am J Roentgenol 2005;185:1531-9.

Cite this article as: Liu B, Zhang Y, Gong J, Jiang S, Huang Y, Wang L, Xu J, Qiu C. CT findings of pulmonary nocardiosis: a report of 9 cases. J Thorac Dis 2017;9(11):4785-4790. doi: 10.21037/jtd.2017.09.122 